\title{
Representaciones estereotípicas del espacio fronterizo en algunas ficciones mexicanas sobre Tijuana
}

\section{Stereotypical Representations of the Border Space in some Mexican Fictions about Tijuana}

\section{Resumen}

La ciudad de Tijuana, arquetipo por excelencia para pensar las dinámicas sociales y culturales de la vida en la frontera, ha sido descrita y representada hasta el cansancio en la literatura. Ciudad de paso sin cultura ni pasado, laboratorio posmoderno equiparable a Nueva York, y capital internacional del crimen organizado son algunos de los lugares comunes frecuentes en la literatura de y sobre Tijuana. Este trabajo revisa algunas ficciones de autores bajacalifornianos como Gabriel Trujillo Muñoz, Heriberto Yépez y Luis Humberto Crosthwaite para deconstruir los prejuicios y estereotipos más comunes en torno a la singular ciudad fronteriza mexicana.

Palabras claves

Tijuana, Frontera, Gabriel Trujillo Muñoz, Heriberto Yépez, Luis Humberto Crosthwaite

\begin{abstract}
The city of Tijuana, the quintessential archetype for thinking the social and cultural dynamics of life on the border, has been described and represented to the point of exhaustion in literature. Tijuana as a transit point without culture or past, Tijuana as the laboratory of postmodernity and Tijuana asthe international capital of organized crime are some of the frequent common places in the literature about Tijuana. This article reviews some fictions of Californians authors such as Gabriel Trujillo Muñoz, Heriberto Yépez and Luis Humberto Crosthwaite to deconstruct the most common prejudices and stereotypes around the unique Mexican border city.
\end{abstract}

Keywords 
CATEDRAL TOMADA: Revista de crítica literaria latinoamericana / Journal of Latin American Literary Criticism Representaciones estereotípicas del espacio fronterizo en algunas ficciones mexicanas sobre Tijuana

Tijuana, Borderline, Gabriel Trujillo Muñoz, Heriberto Yépez, Luis Humberto Crosthwaite

La ciudad de Tijuana es, sin duda, un espacio sobre-representado. Artículos académicos de diversas disciplinas, películas y series de televisión, novelas y corridos musicales se detienen una y otra vez en la urbe fronteriza para celebrar, criticar o simplemente describir las prácticas sociales, culturales y económicas que convergen en la ciudad emplazada en los límites políticos entre ambos países. En el caso específico de la literatura, en las últimas décadas hemos sido testigos de un esfuerzo sostenido lo mismo por las instituciones culturales que por el mercado, por legitimar la existencia de una literatura fronteriza con rasgos propios. Por dar solo un ejemplo de este fenómeno, baste con mencionar el Premio Binacional de Novela Joven Frontera de Palabras / Border of Words, fundado en el año 2001 por el CONACULTA a través del Fondo Cultural Tierra Adentro, el Centro Cultural Tijuana y la Secretaría de Relaciones Exteriores. Dicho premio se convoca de forma anual y se dirige a "los escritores mexicanos residentes en los estados fronterizos de México (Baja California, Sonora, Chihuahua, Coahuila, Tamaulipas y Nuevo León) y de Estados Unidos (California, Texas, Arizona, y Nuevo México)". ${ }^{1}$ Lejos de cuestionar la existencia de una literatura fronteriza diferente y diferenciadora a lo que por oposición llamaríamos una literatura "del centro", en las próximas páginas quisiera analizar desde el espacio algunas representaciones de la frontera méxico-estadounidense presentes en la literatura. ¿Quiénes escriben sobre la frontera?, ¿en qué elementos, prácticas y prejuicios se detienen los narradores y ensayistas al describir la vida fronteriza? Esta pregunta es clave, pues como bien afirma Sayak Valencia

toda aproximación sobre Tijuana debe dialogar con -y al mismo tiempo desafiar- los tres clichés más comunes sobre la ciudad: Tijuana, laboratorio de la posmodernidad; Tijuana, ciudad de paso, y Tijuana,

\footnotetext{
${ }^{1}$ Disponible en http://www.elem.mx/institucion/datos/1680
} 
ciudad de vicio $^{2}$, sin que por ello tengan que ser obviados, pues hay que reconocer que dichos elementos perviven en la ciudad y forman parte importante de ella. Sin embargo, por sí mismos no explican la realidad que conforma una frontera tan contradictoria. (138)

Existen, en efecto, una serie de estereotipos en torno a la frontera en general y a la ciudad de Tijuana en particular que insisten en reducir y explicar todas las dinámicas sociales, culturales y económicas desde ideas preconcebidas arraigadas en el inconsciente colectivo de los mexicanos. El objetivo de este trabajo es doble: por un lado refiere a los principales discursos ensayísticos que han dado origen a dichos estereotipos (ciudad de paso, laboratorio de la posmodernidad, y ciudad de vicio), para posteriormente revisar cómo los novelistas parodian, reconfiguran o desmienten dichos lugares comunes desde la ficción. Es decir, me interesa analizar cómo algunos escritores bajacalifornianos recuperan los discursos preexistente en torno a la ciudad de Tijuana para establecer un diálogo con el (los) cliché(s).

El corpus literario de y sobre la frontera es inabarcable. Por consiguiente, en las próximas páginas tomaré algunos ejemplos concretos de los escritores bajacalifornianos más relevantes para revisar ciertos discursos específicos en torno a las prácticas de espacio propias de la frontera. Me refiero a Gabriel Trujillo Muñoz, Heriberto Yépez, Luis Humberto Crosthwaite y Federico Campbell. La elección no es arbitraria; dichos autores han asumido la frontera como su lugar de enunciación, destacándose como la primera línea de la literatura bajacaliforniana. De igual modo, las obras analizadas a continuación no sólo se hacen cargo de los clichés en torno a la ciudad sino que, como bien apunta Sayak Valencia, dialogan y desafían a los mismos. De esta manera, los principales discursos en torno a la ciudad serán revisitados, reconfigurados y en su caso exagerados hasta el grotesco por las principales plumas bajacalifornianas.

Un buen punto de partida para acercarse a los discursos en torno a la frontera es la zaga policial de Miguel Ángel Morgado creada por Gabriel Trujillo Muñoz.

\footnotetext{
${ }^{2}$ Cursivas del autor.
} 
El ensayista y novelista en repetidas ocasiones ha retratado las ciudades de Mexicali y Tijuana a través de un singular detective, el defensor de los derechos humanos Miguel Ángel Morgado. Dentro de esta zaga quisiera detenerme por ahora en la noveleta Tijuana City Blues. En esta obra, como bien señala Miguel Rodríguez Lozano, "el interés se dirige al conocimiento de la historia local" (70) siendo la trama policiaca una excusa para organizar una búsqueda en torno a las principales "ideas alrededor de esa ciudad" (70). Y aquí es donde surge, precisamente, el primer gran prejuicio en torno a Tijuana:

Para Morgado, Tijuana era un puente, una fila de autos, el paso inevitable rumbo al Parque Balboa y Sea World, en San Diego. Una parte de su infancia. La de las vacaciones de Semana Santa o de verano. Las conocidas excursiones familiares en la camioneta Plymouth destartalada, otro clima. La brisa del mar. La playa. La vista panorámica de la isla Coronado [...] Pero eso era San Diego. Tijuana era un manchón de cerros y calles serpenteantes en su memoria. Mucha gente de compras. Muchas tiendas de segunda. Anuncios de neón gigantescos y calles lodosas. Gente que gritaba sus gangas y se encaramaba a la camioneta de su padre con juguetes japonenses que no funcionaban ni una semana. (Trujillo Muñoz, 175-176)

Tijuana, tal como el protagonista la recuerda, tiene entonces la marca de lo pasajero y lo desgastado; es el purgatorio de mercancías piratas, lodo y neón por el que es necesario atravesar para llegar al paraíso norteamericano. La representación de Tijuana presente en la novela niega de antemano a la ciudad cualquier posibilidad de participar de la cultura nacional o de desarrollar una cultura propia quedando así reducida a una simple estación de paso. Un dato cargado de significación: el personaje de Miguel Ángel Morgado es oriundo de Mexicali, ciudad que abandonó en la adolescencia para residir en lo sucesivo en Ciudad de México. El viaje de regreso a la frontera tiene entonces una significación especial 
pues ofrece la posibilidad de cotejar la ciudad recordada con la ciudad efectivamente encontrada en el presente. El resultado de este ejercicio es negativo: antes de volver a la Ciudad de México, Morgado ofrece al lector una última apreciación sobre Tijuana:

Morgado contempló los cerros cubiertos de casas de lámina y autos inservibles, la hilera dispareja de llantas usadas que servían de escalinatas, los muros con pintas políticas y buenos deseos de prosperidad y democracia. El rostro verdadero, multitudinario, de Tijuana, "La espantosa", como Aidé la llamaba, estaba ahí, a la vista de todos. Sin maquillaje ni lentejuelas. (214)

El viaje a la ciudad vagamente evocada a través de los recuerdos de la infancia no desmiente la imagen negativa sobre Tijuana; antes bien la confirma. Sin embargo, es justo reconocer que antes de viajar a la frontera, Morgado ya había sido predispuesto en contra de la ciudad. En efecto, a los prejuicios de Aidé se suman los de Fernando Jordán cuyo ensayo El otro México, biografía de Baja California (1951), el abogado y detective lee durante el trayecto a la frontera. Así, los comentarios peyorativos de su amiga Aidé, las descripciones nada favorables de Fernando Jordán y sus propios recuerdos negativos, anulan de ante mano la mirada neutral y "objetiva" del espacio. Usando la acertada expresión de Marco Kunz, "la mala reputación eclipsa la experiencia directa, el mito se impone a la realidad" (259). Por contraposición con la urbe fronteriza, la Ciudad de México -a la que pertenece Morgado en el presente narrativo- con sus ruinas prehispánicas, templos barrocos y edificios modernos de acero y cristal implícitamente se erige como la sede de la "verdadera" cultura mexicana. Bajo este punto de vista a todas luces centralizador, avanzar hacia el norte del país implica la paulatina pérdida lo "nacional", siendo inevitable que al llegar a la frontera ya sólo queden los desechos de lámina, los neumáticos utilizados como escaleras, y las baratijas japonesas que no duran más de una semana. 
La imagen de Tijuana que ofrece Gabriel Trujillo Muñoz es todo menos única. Por dar otro ejemplo, el célebre ensayista y narrador Heriberto Yépez en una novela titulada Al otro lado (2008) perpetúa el estereotipo. Aunque la novela se ambienta en una ficticia urbe llamada Ciudad de Paso, ciertas referencias explícitas muy pronto llevan al lector a la conclusión que el espacio no es otro que la ciudad de Tijuana. Por ejemplo, Heriberto Yépez centra la vida nocturna de Ciudad de Paso en una calle llamada Nueva Revolución, lo que supone una referencia muy clara a la conocida Avenida Revolución de Tijuana. Así, el espacio en la novela debe ser visto desde una doble posibilidad: como una lectura concreta de la ciudad de Tijuana, y por antonomasia como una descripción general de la vida en la frontera. Cuando hablamos de Tijuana hablamos de toda la frontera, pues en la imaginación popular esta ciudad de algún modo concentra, engloba y exacerba todas las propiedades que se le atribuyen a la franja fronteriza. Por consiguiente, la avenida Nueva Revolución de Heriberto Yépez puede ser la avenida Revolución de Tijuana y al mismo tiempo cualquier otra calle en cualquier otra ciudad fronteriza. En cualquier caso la representación del espacio que ofrece Yépez guarda importantes semejanzas con la ciudad evocada y visitada por Morgado:

Ahí está el infiernito, el Fracaso Bonito, El Regreso al Pollo Feliz o el King Kong [...] Ahí escuchas banda, tambora, música norteña y sureña, para los nostálgicos que no han perdido los chúntaros. Y para los más jóvenes, música en inglés de todas las épocas y sabores. Ahí, pues, las rockolas son el revoltijo de ritmos. No es extraño ver a un mariachi, en su hora de descanso, echarse una cerveza californiana y poniendo en la rockola, gansta rap, "para variarle".

Sin embargo, era evidente que el ambiente estaba mucho más intenso en la Nueva Revolución, y sus licorerías, farmacias y tiendas de Curious en los primeros pisos y en los segundos todo repleto de antros para güeros, inmigrados y negros; la música techno salía de las terrazas más exclusivas y la música negra de los antros para hip hoperos. Música inmigrada no se 
escuchaba esta noche, ni se escucha nunca aquí, porque los inmigrados no han podido inventar sus propios ritmos ni finos ni callejeros. Para lo único que no han sido lentos es para el resentimiento. $(49,50)$

Ciudad de inmigrantes, conglomerado de cantinas y centros nocturnos, simple estación de tránsito por la que hay que atravesar para llegar al paraíso prometido al otro lado, Ciudad de Paso es un gran collage que reúne elementos disimiles y diversos de México (tambora, música norteña y sureña) y de los Estados Unidos (música en inglés de todas las épocas, techno, hip hop y gansta rap). Hablamos de reunión, no de asimilación o transculturación porque los inmigrantes que conforman el grueso de la población urbana no son capaces de crear sus propios ritmos finos o callejeros, sólo saben repetir y celebrar las músicas mexicanas creadas al sur de la frontera y/o las norteamericanas al norte. Y es justamente en la ausencia de una cultura propia y en la explotación comercial del vicio, representada la primera a través de la música y la segunda en los múltiples bares y cantinas, que emerge el resentimiento como lo único genuino y propio de la frontera. Un resentimiento que crece y se expande tan rápido como las barriadas improvisadas en los cerros de la ciudad.

En un primer acercamiento, Gabriel Trujillo Muñoz y Heriberto Yépez entregan representaciones del todo peyorativas sobre el espacio fronterizo. El desecho y la mugre, las mercaderías baratas y el ruido que surge de la mixtura de músicas ajenas reproduciéndose de manera simultánea en una gran avenida caótica, son al parecer los elementos distintivos de la frontera en general y de Tijuana en particular. Las ficciones, sin embargo, no son tan simples ni maniqueas como parece. Lejos de ofrecer una imagen "fiel" de la frontera (¿acaso es posible representar fielmente un espacio?), ambos novelistas recogen los discursos preexistentes en torno a Tijuana para exhibirlos frente al lector. Dicho con otras palabras, Tijuana City Blues y Al otro lado no pretenden describir objetivamente el espacio fronterizo ni tampoco pretenden construir un discurso "sólido" en torno a las dinámicas sociales y las prácticas culturales de la frontera; el objetivo de ambas 
novelas consiste más bien en recuperar los estereotipos más comunes (como los presentes en el libro de Roberto Jordán) para desmontarlos y exhibir su arbitrariedad. De hecho, Heriberto Yépez en un divertido y revelador ensayo publicado en el 2006, postuló el término “Tijuanologías" para referirse no la ciudad real de Tijuana sino a su invención a través del discurso: para Yépez, Tijuana "más que una ciudad, es una religión o una mitología maldita" (13). Existen tantos discursos en torno a la ciudad, tantas citas, artículos y novelas en torno a ella, que

<<Tijuana〉> no es más que un filosofema. Una entelequia que no existe sino en una colmena de citas y en un apiladero de artículos. Alguien empezó a discutir Tijuana en busca de su esencia y su sucesor se tragó el cuento de que tal entidad existía realmente y así ya van varias generaciones intertextuales que se proponen discutir la esencia de una supuesta ciudad mítica llamada Tijuana, que en realidad no es más que una frontera imaginaria. (Yépez 118)

Frente a los múltiples estereotipos en torno a la frontera, Yépez renuncia de ante mano a describir la Tijuana "real" y prefiere jugar con la ambigüedad . Ciudad de Paso es y no Tijuana, pues los males que se la adjudican a la ciudad bajacaliforniana son obsérvales en cualquier otra urbe contemporánea. Como bien señala Marco Kunz,

Ante la imposibilidad tanto de definir Tijuana como de erradicar los estereotipos sobre ella, Yépez optó por construir con estos últimos una versión ficticia de la frontera que supera en horror y absurdo las más horripilantes fantasmagorías de la tijuanología distópica y le muestra a ésta la fiel imagen de su desmesura. (Kunz 268)

Bajo este punto de vista, el hecho de que Yépez renuncie a nombrar directamente la ciudad y prefiera nombrarla como Ciudad de Paso se entiende desde 
una bien intencionada propuesta estética: Al otro lado no es una novela sobre Tijuana sino sobre la "tijuanología": es decir, sobre los estereotipos grotescos y desmesurados en torno a Tijuana, pues al nombrar la ciudad con otro topónimo Yépez rompe con el pacto mimético y se libera de un realismo que seguramente hubiese desembocado en la repetición de los estereotipos. Ciudad de paso no repite entonces los estereotipos; los lleva más lejos, los explota y los deforma hasta volverlo inverosímiles.

Volvamos por ahora a la novela de Trujillo Muñoz para recordar que antes de viajar a Tijuana, el detective Morgado recoge ciertas apreciaciones desfavorables de la ciudad principalmente a través del ensayo de Fernando Jordán. Añadamos ahora que una vez instalado en la ubre fronteriza, el protagonista absorbe otros discursos en torno a ella que se sitúan en las antípodas de las opiniones expresadas por Jordán. Me refiero al profesor Leobardo, historiador y cronista de la ciudad para quien Tijuana es algo así como la utopía efectivamente realizada:

El norte no es lo que él [José Vasconcelos] decía. No somos, ¡de ninguna manera!, la cultura de la carne asada y la tortilla de harina. También somos sensibilidad y pensamiento. Somos un México nuevo, el futuro de la nación mexicana, el espejo modernizador de la patria. Tijuana es el símbolo más preclaro del México del siglo venidero. Sólo es necesario que el resto de los mexicanos se quite las anteojeras del prejuicio y vea lo que realmente somos: una ciudad-crisol de razas y pueblos, una metrópoli que avanza, marchas forzadas, hacia un porvenir impoluto y diamantino [...]. $(185)$

El entusiasta discurso de Leobardo es todo menos original. Así como existe una imagen estereotípica de Tijuana que insiste en definirla como una gran fayuca sin pasado ni cultura, existe igualmente un contra-discurso que celebra la ciudad precisamente por su capacidad para absorber lo diverso y anticiparse al futuro. Pienso, por ejemplo, en el célebre estudio de Néstor García Canclini Culturas 
híbridas, estrategias para entrar y salir de la modernidad (1990), en donde el ensayista dedica un apartado para describir las prácticas sociales de la ciudad. De acuerdo con Canclini, Tijuana ha abandonado su condición de periferia para convertirse "junto con Nueva York, [en] uno de los mayores laboratorios de la posmodernidad" (293). En efecto, si uno de los rasgos constitutivos de la posmodernidad consiste en la disolución de los cuerpos sociales tradicionales como la familia, la iglesia, las asociaciones cívicas y, en síntesis, el pueblo como un cuerpo social unificado a partir de una supuesta homogeneidad de elementos como la lengua, la raza, la ideología y la religión, la frontera norte de México deviene por excelencia en la representación máxima de lo posmoderno. Pues en Tijuana, siempre siguiendo a Canclini, "los oficios se toman y se cambian con la misma versatilidad que los coches y las casas" (293). El bilingüismo, la transitoriedad de las relaciones laborales, la posibilidad de trabajar y/o estudiar en los Estados Unidos sin dejar de vivir en México, y el indistinto uso de dólares y pesos suponen un ejercicio constante de hibridación que sitúa a los sujetos fronterizos como pioneros en un mundo en vías de transformarse en un gran no-lugar. La sociedad líquida de Zygmunt Bauman es ya una realidad en la frontera norte de México, desarticulando por completo la ficción del "pueblo" como un cuerpo social homogéneo. Bajo este punto de vista no está tan alejado de la verdad el profesor Leobardo cuando eleva a Tijuana al estatuto del "símbolo más preclaro del México venidero"; un México en donde lo local terminará por diluirse en lo global, quedando tan sólo una mezcolanza caótica de símbolos culturales disímiles pero liberados de cualquier peso atávico.

El narrador Luis Humberto Crosthwaite en Estrella de la calle sexta (2000) propone una metáfora por demás sugestiva del celebrado hibridismo cultural a través de un singular personaje que pasa las noches del sábado en una famosa esquina del centro de la ciudad. Alcohólico, poeta y amante ocasional, El Güero, protagonista del cuento "Sabaditos en la noche", es un personaje camaleónico: a pesar de ser mexicano todos lo toman por gringo por su color claro de piel y su cabellera rubia, su verborrea combina el castellano con el inglés, y su conducta 
misma combina la fascinación por la calle y la multitud del flâneur con la pasividad del voyeur. Es verdad que El Güero frecuenta algunos bares y hoteles de la calle sexta, pero más que caminar la calle se instala en ella hasta casi convertirse en parte del mobiliario urbano, por lo que no termina de ser el flâneur benjaminiano: "nada como estar sentado tanto tiempo que ya eres parte de la banqueta, igual que un semáforo"(62), señala el propio Güero. Y es justamente en esta obsesión por permanecer en el espacio en lugar de transitar por él que en la mirada de Crosthwaite, Tijuana deja der ser una simple ciudad de paso para adquirir una densidad propia ${ }^{3}$. La frontera puede, en efecto, ser una simple estación de paso para los hispanos que buscan avanzar al norte o bien una enorme cantina para los norteamericanos que buscan fiesta y alcohol al sur. Sin embargo, la frontera es también el hogar permanente de millones de personas. No todos son turistas o pasajeros en tránsito en la frontera, la población flotante es parte del tejido social pero no puede, de ninguna manera, definir el total de las prácticas sociales y culturales. De ahí que Luis Humberto Crosthwaite se esfuerce por buscar lo "propio" desde un singular personaje que decide permanecer y arraigar en la esquina antes que en transitar por la avenida.

Ahora, lo más sugerente del personaje de Crosthwaite reside en su oficio de carrocero de automóviles "dedicado a hacer que las cosas sean como fueron, capaz de borrar las huellas de los accidentes, devolver el pasado" (60). Su obsesión por reparar carrozas remite a un evento biográfico por demás traumático; el accidente automovilístico en el que perdió a su familia. Pero si salimos del plano biográfico para interrogar el gusto por el oficio como una metáfora de la frontera en general y de Tijuana en particular, se perfila una visión muy sugerente sobre la posibilidad de construir una identidad nacional libre de cargas atávicas. La frontera es acaso el único espacio en el que el sujeto puede verdaderamente reconfigurarse borrando los traumas del pasado tal como el carrocero elimina en las superficie de los automóviles las huellas de los accidentes. Porque El Güero no niega el pasado; lo restaura, lo libera de su carga negativa. El movimiento incesante de la frontera y la

\footnotetext{
${ }^{3}$ Véase el sugerente ensayo de Elena Ritondale presente en la bibliografía.
} 
hibridación de códigos y prácticas culturales diversas ofrece la posibilidad de actuar el pasado sin dejar de interpretar en paralelo otros roles. El personaje de Crosthwaite es una especie de antihéroe posmoderno abierto a múltiples referencias culturales que lejos de excluirse o entrar en tensión las unas con las otras, logran una especie de síntesis provisional. En la frontera el pasado más que repetirse así mismo en una franca negación al cambio, se actualiza e hibrida con el presente y el futuro.

Esta mirada laudatoria en torno a la frontera y sus prácticas culturales no deja de ser tan peligrosa y engañosa como su contraparte previamente revisada; es decir, la ciudad sin pasado, cultura e identidad. Como bien señala Sayak Valenciana en Capitalismo gore, celebrar la frontera por su hibridación

nos remite a una lectura dolosa, reducida y celebratoria que integra las polaridades a manera de lego que puede montarse y desmontarse, pero que está lejos de considerar las verdaderas implicaciones físicas que recaen sobre los cuerpos a través de sus condiciones socioeconómicas y geopolíticamente situadas. (Valencia 136)

Con estas palabras entramos de lleno en un nuevo discurso sobre la frontera que igualmente podemos detectar y deconstruir desde la literatura. Me refiero a la representación de la frontera como una enorme zona geográfica sin ley ni Estado y como tal propensa a la celebración y la práctica de todas las actividades delictuales posibles. Sobre el ensayo de la tijuanense Sayak Valencia hablaremos a continuación; antes quisiera volver por un momento a la novela de Trujillo Muñoz, Tijuana City Blues: ya ha sido señalado que el protagonista, el detective Morgado, durante su visita a la frontera absorbe distintos discursos en torno a la ciudad; Tijuana como un espacio de tránsito caótico y desprovisto de cultura, y al mismo tiempo como una ciudad privilegiada para practicar la sociedad líquida posmoderna y como tal anticipar el futuro de todo México. Agreguemos ahora un tercer lugar común: la frontera como la máxima sede trasnacional del crimen organizado. 
- Me han asegurado que ya es una ciudad en forma, un centro industrial y de negocios-acotó Morgado-; la ciudad del futuro.

- Sí. Pero no te han dicho qué clase de industrias son las más prósperas. Te diré yo algunas: las del espionaje industrial y la de los desechos tóxicos. Y si hablas de negocios, allí están los de las religiones y los cultos a cualquier cosas, desde la iglesia del extraterrestre crucificado hasta la del vampiro cósmico. Otros business que compiten por el primer puesto son los del tráfico de órganos, el contrabando de armas y la contratación de sicarios (si no pregúntale al cardenal Posadas), y los más venerables de todos: los de los polleros, los del narcotráfico y los de la prostitución a escala masiva. Todo eso hace que Tijuana sea un factor de peso en el mercado de capitales a nivel mundial (198).

Estas palabras pronunciadas por Harry Dávalos, un agente del FBI con el que colabora Morgado con cierta frecuencia, sintetizan una visión ampliamente compartida en torno a la frontera. De hecho, sólo una década después de la publicación de la novela de Trujillo Muñoz, Sayak Valencia sorprendió a la academia internacional con un lúcido ensayo sobre el estado actual del capitalismo en las sociedades contemporáneas. Un capitalismo in extremis que utiliza, crea y propaga la violencia como una estrategia utilizada por diversos grupos marginales para acceder al consumo. De ahí que el término empleado por Valencia sea el de Capitalismo Gore en franca alusión un tipo específico de cine en donde la violencia es explícita y la sangre aparatosa. Un término que en el caso específico de Tijuana busca además recoger los tres principales clichés sobre la urbe (ciudad de paso, laboratorio de la posmodernidad y ciudad de vicio) para reinsertarlos a través de "uno de los epicentros fundamentales de la ciudad: su economía de la violencia" (Valencia 138). Aunque las prácticas descritas por Valencia son universales y podemos localizarlas en cualquier parte del orbe, la ensayista reserva para Tijuana el epítome de capital gore. 
De acuerdo con Valencia la frontera norte de México es un territorio especialmente fértil para la emergencia de grupos de delincuencia organizada que mediante la violencia desplazan al Estado para instaurar un orden social y económico en el que desaparecen los contornos entre lo legal y lo ilegal. Así, mientras Luis Humberto Crosthwaite y Néstor García Canclini celebran la frontera por su hibridismo cultural y por su capacidad para montar y desmontar polaridades, Sayak Valencia define la frontera como zonas nacionales de sacrificio en

donde se instauran dinámicas dobles que hacen de dichos territorios un espacio donde todo vale; es decir, se las considera el garaje de los dos países. Territorios- puerta, backdoor cities, en los que confluyen de la misma manera y, simultáneamente, lo indeseable y lo deseable, hibridando estas características y haciendo difícil la aplicación de una axiología tradicional para su conceptualización, creando una especie de ruptura escatológica desde la cual se las concibe como autófagas y siniestras. (134135)

El crimen es universal, pero en Tijuana se industrializa, se propaga y se normaliza hasta formar parte sustancial de las dinámicas y prácticas sociales. Más aún, pareciera anular por completo cualquier actividad legal para instaurar el delito como la única actividad social y económica posible a través de la comercialización masiva y sistémica del vicio. De ahí que Heriberto Yépez construya una ficción exagerada utilizando como materiales el tráfico de personas y de drogas, y la violencia irracional de los jóvenes sicarios adictos al phoco (droga sintética elaborada con cocaína, veneno para ratas y la tierra de los cerros de Ciudad de Paso). Puede, desde luego, objetarse que estos elementos no son propiedad exclusiva de Tijuana. Sin embargo, la imaginación popular insiste en focalizar dichas prácticas sociales en la frontera mexicana, principalmente en ciudades como Tijuana y Ciudad Juárez, como si el resto del país fuese un oasis de paz frente a la barbarie fronteriza. Al respecto, los ejemplos literarios abundan. Por dar sólo unos 
casos, en la excelente pero extrañísima novela Todo lo de las focas (2005) el bajacaliforniano Federico Campbell otorga la siguiente descripción sobre Tijuana:

La ciudad se fue extendiendo hacia los cerros, vivía de contrabando de leche y gasolina, llantas y accesorios de automóvil, se barrían los dólares con escoba, su población flotante dejaba de serlo en cuanto terminaban las guerras, y así, de una ranchería de finales de siglo pasó a ser un pueblo fantasma al principio, luego una maravillosa tierra de nadie en la que tanto los visitantes como los nativos se sabían perdidos y sólo fraguaban negocios de remuneración inmediata y aspiraban a industrializar el aborto, los juegos de azar, los centros de diversión y las baratijas artesanales. (36)

La novela es una historia de amor contada desde la mirada de un narrador nostálgico que evoca en retrospectiva distintos episodios de su pasado. La mujer amada tanto como el espacio fronterizo tienen la marca de lo onírico, pues el recuerdo y la fantasía parecen enredarse y confundirse siendo imposible deslindar los límites entre lo real y lo soñado. Así, mientras las imágenes de Beverly dotan a la novela de una atmosfera erótica y poética, la descripciones sobre la ciudad ofrecen un sórdido contraste a través de la evocación permanente a las economías del crimen y comercialización del vicio. La frontera se convierte entonces en un espacio polisémico que demarca los límites políticos entre ambos países, entre el espacio onírico y la vigilia, y por último entre el deseo representado en Beverly y por sinécdoque en los Estados Unidos y el horror escenificado en Tijuana y su economía del crimen.

Recapitulando, la frontera norte de México en general y la ciudad de Tijuana en particular han sido objeto de múltiples discursos. Hay un creciente interés por describir la frontera, por definir sus prácticas de espacio y volverlas inteligibles a través del discurso. Ciudades sin historia ni cultura habitadas por migrantes y dedicadas a la explotación comercial del vicio, laboratorios de la posmodernidad y capitales internacionales del crimen organizado (capitalismo 
gore). ¿Cuál es entonces la "imagen real” de la frontera mexicano-estadounidense? ¿La(s) horribles ciudades de paso dedicadas a la industria de los excesos que en su momento describió Fernando Jordán? ¿El gran laboratorio posmoderno que en su momento celebró Néstor García Canclini? ¿La capital mundial del Capitalismo Gore como propone Sayak Valencia? La literatura de y sobre la frontera mexicana mantiene una relación ambivalente con estos discursos estereotípicos. Por momentos, los escritores parecieran coincidir con ellos y proponen sus propias metáforas para confirmar el estereotipo en turno, como ocurre por ejemplo con Luis Humberto Crosthwaite y su antihéroe El Güero quien de algún modo plasma poéticamente la visión fronteriza de García Canclini. Por momentos, la literatura paciera también alejarse del estereotipo mediante representaciones paródicas, grotescas y exageradas, como ocurre con Heriberto Yépez respecto a las lecturas peyorativas sobre la ausencia de cultura y la masificación del crimen en la frontera. Y por momentos la literatura paciera simplemente recoger los principales discursos sobre la ciudad para presentarlos ante el lector y exhibir su arbitrariedad como ocurre en las novelas de Trujillo Muñoz y Federico Campbell respectivamente. En cualquier caso, las ficciones no pueden leerse al margen de los múltiples discursos ensayísticos previos sobre la frontera pues en cada caso se establece un diálogo particular entre el prejuicio y el espacio real y tangible.

Gabriel Trujillo Muñoz en un ensayo titulado "Conflictos y espejismos; la narrativa policiaca fronteriza mexicana", señala que las primera representaciones de Tijuana presentes en la literatura fueron el resultado de la visión de escritores del centro del país como Paco Ignacio Taibo II y Juan Hernández Luna. Esta "extranjería" con respecto a la frontera acaso explica que el lugar común eclipse la experiencia directa, retomando la expresión de Marco Kunz. De acuerdo con Trujillo Muñoz

Los beneficios y maleficios de vivir en la frontera ya son el sello distintivo de la vida comunitaria en el resto del país. Lo que ahora aparece, ya en los últimos años, es la novela policiaca fronteriza escrita desde el corazón 
del monstruo, como testimonio existencial y crónica diaria. Un trazo de luz, de lucidez, en la tiniebla prevaleciente. (Trujillo 36)

En efecto, a estas alturas del partido sería un acto de necedad seguir encasillando las mercaderías piratas, las chabolas de cartón y lámina con neumáticos ensamblados a manera de escalinatas, los prostíbulos y los bares, y la violencia y el narcotráfico como prácticas privativas de la frontera. De ahí que novelas como Tijuana City Blues y Al otro lado no pretendan otorgar una imagen definitiva sobre Tijuana en particular o la frontera en general. El objetivo de ambas novelas apunta más bien a la recolección de discursos y lugares comunes para mostrar los excesos en las representaciones preexistentes.

Los múltiples discursos que una y otra vez se han construido en torno a la frontera se sobreponen los unos a los otros creando una "segunda realidad" que al margen del espacio físico y palpable pareciera existir sólo en y a través del lenguaje. Las obras narrativas aquí revisadas no pueden entenderse al margen de los estereotipos preexistentes en torno a Tijuana. En efecto, Gabriel Trujillo Muñoz, Heriberto Yépez, Luis Humberto Crosthwaite y Federico Campbell recuperan, desafían, cuestionan o exageran los lugares comunes dotando a sus textos de una complejidad discursiva que no debería pasarse por alto o tomarse a la ligera. ¿Cuál es entonces la verdad sobre la frontera? Acaso la mejor respuesta, que de hecho no responde nada por paradójico que esto parezca, es que las fronteras y sus prácticas son tan indefinibles como cualquier otro espacio del orbe. 
CATEDRAL TOMADA: Revista de crítica literaria latinoamericana / Journal of Latin American Literary Criticism Representaciones estereotípicas del espacio fronterizo en algunas ficciones mexicanas sobre Tijuana

\section{Bibliografía}

Campbell, Federico. Todo lo de las focas. Universidad de Guanajuato, 2005.

Crosthwaite, Luis Humberto. Estrella de la calle sexta. Tusquets, 2016.

García Canclini, Néstor. Culturas híbridas. Estrategias para entrar y salir de la modernidad. Debolsillo, 2009.

Jordán, Fernando. El otro México. Biografía de Baja California. Universidad Autónoma de Baja California, 1993.

Kunz, Marco. "Tijuana la indefinible. Narcorrealismo y esperpento en la obra de Heriberto Yépez". Nuevas Narrativas Mexicanas, edited by Marco Kunz, Mondragón, Phillipps, 2012, pp. 259-271. Red ediciones

Ritondale, Elena. "Ni ciudad maldita ni no lugar. La Tijuana fronteriza de Luis Humberto Crosthwaite". Dimensiones. El espacio y sus significados en la literatura hispánica, edited by Raquel Crespo-Villa, Pastor, 2017, pp. 53-60. Biblioteca Nueva.

Rodríguez Lozano, Miguel. "De fronteras asediadas. Sobre El festín de los cuervos de Gabriel Trujillo Muñoz". El norte y su frontera en la narrativa policiaca mexicana, edited by Juan Carlos Rámirez Pimienta, Fernández, 2005, pp.61-77. Plaza y Valdés.

Trujillo Muñoz, Gabriel. "Tijuana City Blues". El festín de los cuervos. La saga fronteriza de Miguel Ángel Morgado, 2002, pp. 145-217. Norma, 2002. . "Conflictos y espejismos. La narrativa policiaca fronteriza mexicana". El norte y su frontera en la narrativa policiaca mexicana, edited by Juan Carlos Rámirez Pimienta, Fernández, 2005, pp.61-77. Plaza y Valdés.

Valencia, Sayak. Capitalismo gore. Control económico, violencia y narcopoder. Paidós, 2016.

Yépez, Heriberto. Al otro lado. Planeta, 2008. 
CATEDRAL TOMADA: Revista literaria latinoamericana / Journal of Latin American Literary Criticism Felipe Oliver

. Tijuanologías. Universidad Autónoma de Baja California/ Libros del Umbral, 2006. 